\title{
Bioanalysis
}

\section{HRMS or HRAMS?}

\author{
"...mass resolution can be defined in a mass spectrum as "the observed \\ $\mathrm{m} / \mathrm{z}$ value divided by the smallest difference $\Delta(\mathrm{m} / \mathrm{z})$ for two ions that \\ can be separated: $(\mathrm{m} / \mathrm{z}) / \Delta(\mathrm{m} / \mathrm{z})^{\prime}$."
}

First draft submitted: 31 May 2016; Accepted for publication: 27 June 2016; Published online: 20 July 2016

\begin{abstract}
Keywords: accurate mass $\bullet$ high-resolution MS $\bullet$ mass accuracy $\bullet$ time-of-flight mass spectrometer
\end{abstract}

High-resolution MS (HRMS) continues to get a lot of interest and attention from scientists involved in bioanalytical studies as well as scientists who are working on metabolite identification studies. MS instrument vendors continue to improve the capabilities of their products and bioanalytical scientists continue to look for ways to apply new MS systems to challenging assays in both the drug discovery and the drug development arenas.

Resolution is a well-defined term in MS. As stated in the definitive article by Murray et al., mass resolution can be defined in a mass spectrum as 'the observed $m / z$ value divided by the smallest difference $\Delta(\mathrm{m} / \mathrm{z})$ for two ions that can be separated: $(m / z) /$ $\Delta(m / z){ }^{\prime}[1]$. As a general rule, a mass spectrometer with mass resolution of 10,000 or higher is defined as high resolution $[2,3]$. So, high-resolution mass spectrometers include TOF MS systems as well as Orbitrap MS systems, while quadrupole systems would be called nominal mass resolution systems.

In recent years, we have noted that some publications have referred to HRAMS when describing the use of HRMS systems. While the term HRAMS has been defined as high-resolution accurate MS, we find that definition confusing because it is ambiguous. Should it be read as 'high-resolution-accurate-mass spectrometry' or 'high-resolutionaccurate mass spectrometry'? If it is the former, then what is it describing as accurate?
Does this mean some mass spectrometers are not accurate? If it is the latter, then it would not be preferred because it does not end with MS. In this case, we would argue that what is really meant is 'high-resolution-accurate mass-mass spectrometry' or HRAMMS. We certainly do not want to add to the confusion by proposing HRAMMS as the correct term, so we do not recommend it. Furthermore, we find the terminology, accurate mass, to be misleading. We believe that both low-resolution mass spectrometers and high-resolution spectrometers provide an accurate mass (as long as they are properly calibrated!). The difference, of course, is the number of significant figures in the measurements.

There is a clear terminology in MS that could be applied to this discussion. The term is mass accuracy. Mass accuracy refers to how accurately one can measure the mass of the analyte as compared with its exact mass [2]. Exact mass is the 'calculated mass of an ion or molecule with specified isotopic composition' [1]. It is easier to describe this difference in terms of separation. So, with nominal mass accuracy, one can distinguish an analyte of $520 \mathrm{Da}$ (nominal mass) from an analyte of $521 \mathrm{Da}$ (nominal mass). With high mass accuracy, one can distinguish an analyte of $520.2000 \mathrm{Da}$ (exact mass) from an analyte of 520.2371 Da (exact mass). So, being able to measure the mass with high mass accuracy may allow one to determine the empirical formula of an analyte. This

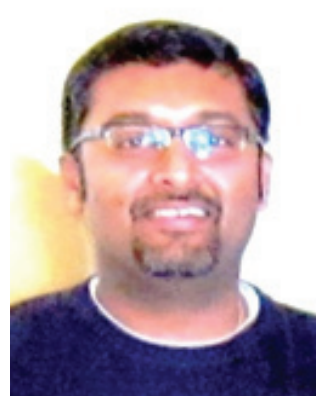

Ragu Ramanathan Author for correspondence: PDM-NCE, Pfizer World Wide Research \& Development, Groton, CT, USA ragu.ramanathan@pfizer.com

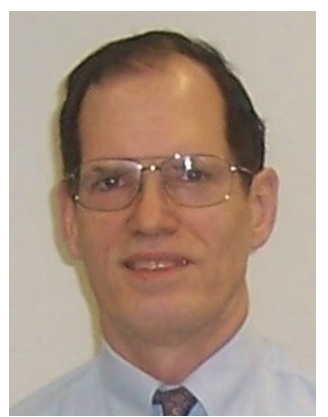

Walter Korfmacher Drug Metabolism \& Pharmacokinetics, Sanofi, Waltham, MA, USA 
can be important when trying to identify an unknown metabolite, for example. Therefore, if we really wanted to properly name MS systems with this capability, then we would need to call them 'high-resolution-high mass accuracy-mass spectrometry' or HRHMAMS. Again, we do not want to add to the confusion by proposing HRHMAMS as the correct term, so we do not recommend it either.

An additional problem with the term HRAMS is the fact that accelerator MS is abbreviated as AMS [4]. This leads to the potential for HRAMS to be misunderstood as high-resolution accelerator MS. So, this is another reason, we feel that HRAMS should be discontinued as an accepted MS term.

We were hoping to use the definitive source by Murray et al. to settle this question [1]. We found it showed many MS-related terms, including 'granddaughter ion,' stating that this 'term is deprecated.' Unfortunately, neither HRMS nor HRAMS made it into this official list. We also tried to find a definition by checking with the American Society for Mass Spectrometry (ASMS) website. While we found no clear guidelines at the ASMS website that help us on this issue, we do note that one of us (R Ramanathan) has been teaching a short course at the annual ASMS conference for multiple years; the course is titled 'High Resolution Mass Spectrometry for Qualitative and Quantitative Analysis: An Introduction.' R Ramanathan also reports that the course has consistently referred to MS applications that use high-resolution mass spectrometers as HRMS applications [5]. A recent review article by Xian F et al. entitled 'High Resolution Mass Spectrometry' included

\section{References}

1 Murray KK, Boyd RK, Eberlin MN, Langley GJ, Liang L, Naito Y. Definitions of terms relating to mass spectrometry (IUPAC Recommendations 2013). Pure Appl. Chen. 85(7), 1515-1609 (2013).

2 Mortishire-Smith R, Zhang H, Bateman KP. High-resolution mass spectrometry and drug metabolite identification. In: Mass Spectrometry in Drug Metabolism and Disposition: Basic Principles and Applications, Lee M, Zhu M (Eds). John Wiley \& Sons, Inc., Hoboken, NJ, USA, 407-448 (2011).

3 Xian F, Hendrickson CL, Marshall AG. High resolution mass spectrometry. Anal. Chem. 84(2), 708-719 (2012). descriptions of various HRMS systems including TOF systems and Orbitrap MS systems as well as applications of 'high resolution MS technology in the fields of food safety...' [3]. As another example, we can cite the recent book chapter by Emary WB, Zhang NR entitled 'Utility of High-Resolution Mass Spectrometry for New Drug Discovery Applications' [6]. The chapter begins with the statement that the 'pharmaceutical industry has used high-resolution mass spectrometry (HRMS) for many years...' The chapter describes the utility of various HRMS systems including both TOF and Orbitrap MS systems.

So, we conclude by stating that we think HRMS systems should be simply called HRMS systems and that the term HRAMS should be discontinued and listed as 'term is deprecated.'

\section{Disclaimer}

R Ramanathan has been teaching a short course titled 'High Resolution Mass Spectrometry for Qualitative and Quantitative Analysis: An Introduction' at the annual ASMS conference for over 10 years [5].

\section{Financial \& competing interests disclosure}

The authors have no relevant affiliations or financial involvement with any organization or entity with a financial interest in or financial conflict with the subject matter or materials discussed in the manuscript. This includes employment, consultancies, honoraria, stock ownership or options, expert testimony, grants or patents received or pending, or royalties.

No writing assistance was utilized in the production of this manuscript.

4 Gao L, Chowdhury S. Pharmaceutical applications of accelerator mass spectrometry. In: Using Mass Spectrometry for Drug Metabolism Studies, Korfmacher W (Ed.). CRC Press, Boca Raton, FL, USA, 391-418 (2010).

5 American Society for Mass Spectrometry. Two-Day Short Courses - Saturday and Sunday, May 30 and 31. www.asms.org

6 Emary WB, Zhang NR. Utility of high-resolution mass spectrometry for new drug discovery applications In: Mass Spectrometry for Drug Discovery and Drug Development, Korfmacher W (Ed.). John Wiley and Sons, Inc, Hoboken, NJ, USA, 37-54 (2013). 